\title{
Electronic specific heats for amorphous and crystallized alloys
}

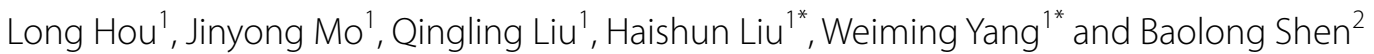

*Correspondence:
liuhaishun@126.com;
wmyang@cumt.edu.cn
${ }^{1}$ School of Sciences, School
of Mechanics and Civil
Engineering, State Key
Laboratory for Geomechanics
and Deep Underground
Engineering, China University
of Mining and Technology,
Xuzhou 221116, China
Full list of author information
is available at the end of the
article

liuhaishun@126.com wmyang@cumt.edu.cn ${ }^{1}$ School of Sciences, School of Mechanics and Civil Engineering, State Key Laboratory for Geomechanics Engineering, China University of Mining and Technology, Full list of author information is available at the end of the provided you give appropriate credit to the original author(s) and the source, provide a link to the Creative Commons license, and indicate if changes were made.

\section{Background}

As a new kind of material, amorphous alloy is known for its promising mechanical (Yang et al. 2014a, b; Dun et al. 2014), magnetic (Liu et al. 2014; Xiang et al. 2014), and chemical (Wang et al. 2012) properties compared to its corresponding crystalline counterpart, and the great differences in properties between the two states are considered to be connected with their ordered or disordered microstructure. Meanwhile, recent study also has revealed that the short- or medium-range order exists at the atomic scale in amorphous alloy (Wang et al. 2008). For the investigation of this interesting atomic structural characteristics and the corresponding mechanism, the low temperature specific heat is considered to be one of the most effective parameters, which is closely linked to the phase transformation (Mikla and Mikla 2009; Xiang et al. 2015), low-energy excitation (Huang 1988; Burin 1995), atomic vibrations (Li et al. 2008), and electronic movement of the solids (Machida and Ichioka 2008). It is known that amorphous alloys can be transformed into crystallized ones by annealing, and obvious changes are induced in the microstructure, accompanied with the variation of the specific heat. So far, for crystallized alloy, the low temperature specific heat can be explained satisfactorily using Debye model, an important theory in solid state physics. On the contrary, for amorphous alloy, although some achievements have been made recently (Blázquez et al. 2008; Kroeger et al. 1984; Kanomata et al. 2008; Grace and Anderson 1989), it still remains a puzzle 
that the specific heat obtained from experiment is usually a little larger than that from theoretical calculation at low temperature, moreover, when $T>1 \mathrm{~K}$, the phonon specific heat, $C_{\text {phonon }}$ still deviates from the expected $T^{3}$ dependence, presenting a broad maximum in $C_{\text {phonon }} / T^{3}$, which is called the "boson peak" (BP) (Zheng 1987; Shintani and Tanaka 2008). Therefore, studying the intrinsic mechanisms of low temperature specific heats for both amorphous and crystallized alloys and exploring the relationship between them are becoming more and more necessary. Zhou et al. (2006) reported that the phonon specific heat at 1.8-154 K can be fitted using Debye model and Einstein oscillators model; Wang et al. (2011) studied the low temperature specific heat of $\mathrm{Cu}_{60} \mathrm{Zr}_{20} \mathrm{Hf}_{10} \mathrm{Ti}_{10}$ alloy and fitted the specific heats of amorphous and crystallized alloys linearly; Our previous work (Hou et al. 2015) also suggested that the low temperature specific heat can be fitted by electronic and phonon contribution terms. However, these studies mainly focus on the effect of crystallization on phonon specific heat, which induces the change of BP, while the study of crystallization on electronic specific heats for amorphous alloys is very little. At the same time, it has also been concluded that the electronic specific heat is closely related to the structural and mechanical properties (Yang et al. 2013; Yu et al. 2010). Thus, studying the effect of crystallization on the electronic specific heat has important scientific significance, it may disclose the change of electronic density of states and how this change affects the structure of amorphous alloy.

In this paper, from the perspective of electronic specific heat, the electronic contribution term in specific heat for $\left(\mathrm{Fe}_{0.5} \mathrm{Co}_{0.5}\right)_{72} \mathrm{~B}_{20} \mathrm{Si}_{4} \mathrm{Nb}_{4}$ amorphous alloy with the high glass-forming ability (GFA) and a highly random packed microstructure is discussed at low temperature. The result is expected to shed light on the disordered atomic structure in amorphous alloys at low temperature.

\section{Experimental methods}

Multi-component Fe-Co-B-Si-Nb alloy ingots with composition of $\left(\mathrm{Fe}_{0.5} \mathrm{Co}_{0.5}\right)_{72} \mathrm{~B} 20 \mathrm{Si}$ ${ }_{4} \mathrm{Nb}_{4}$ are prepared by arc melting the mixtures of Fe (99.99 mass \%), Co (99.99 mass \%), $\mathrm{Nb}$ (99.99 mass \%) metals, together with B (99.50 mass \%) and Si (99.99 mass \%) crystals in a highly purified argon atmosphere. The alloy compositions represent nominal atomic percentages. The cast bulk $\left(\mathrm{Fe}_{0.5} \mathrm{Co}_{0.5}\right)_{72} \mathrm{~B}_{20} \mathrm{Si}_{4} \mathrm{Nb}_{4}$ amorphous alloy rods are fabricated by a copper-mold casting method (Yang et al. 2013). In the mold casting method, the master alloy is melted in a quartz crucible using an induction coil and pushed thereafter in a copper-mold by applying an ejection pressure of about $0.5 \mathrm{~atm}$. To anneal the sample, the cast rod is cut to pieces $10 \mathrm{~mm}$ in length and placed in silica tube. The silica tube is sealed after evacuated and put into the furnace in vacuum, annealed at $923 \mathrm{~K}$ for $4 \mathrm{~h}$, and then quenched in water. The annealing temperature is higher than the crystallization temperature $\left(T_{\mathrm{x}}=857 \mathrm{~K}\right)$.

Amorphous and crystallized structures are examined by X-ray diffraction (XRD) using a RINT 2000 diffractometer with $\mathrm{Cu} \mathrm{Ka}$ radiation at $40 \mathrm{kV}$. The low temperature specific heats $C_{\mathrm{p}}$ for $\left(\mathrm{Fe}_{0.5} \mathrm{Co}_{0.5}\right)_{72} \mathrm{~B}_{20} \mathrm{Si}_{4} \mathrm{Nb}_{4}$ alloys in cast and crystallized states are measured using the physical property measurement system (PPMS) from quantum design system (Model-9) from 1.4 to $110 \mathrm{~K}$. The final data are obtained by averaging the five experimental results. The relative error for the specific heat measurements is less than $2 \%$. 


\section{Results and discussion}

Figure 1 shows the XRD patterns of $\left(\mathrm{Fe}_{0.5} \mathrm{Co}_{0.5}\right)_{72} \mathrm{~B}_{20} \mathrm{Si}_{4} \mathrm{Nb}_{4}$ alloys in the cast state and crystallized state. Within the scope of the resolution of the XRD, the cast alloy shows a broad diffused peak without the crystallization crystal diffraction peak. This is a characteristic for fully amorphous alloy. After being annealed at $923 \mathrm{~K}$ for $4 \mathrm{~h}$, the crystallized alloy shows many shap diffraction peaks, which indicates the crystallized state formed.

Figure 2 presents $T$-dependent $C_{\mathrm{p}}$ for $\left(\mathrm{Fe}_{0.5} \mathrm{Co}_{0.5}\right)_{72} \mathrm{~B}_{20} \mathrm{Si}_{4} \mathrm{Nb}_{4}$ alloys in the cast state and crystallized state from 1.4 to $110 \mathrm{~K}$. It can be seen that the $T$-dependent $C_{\mathrm{p}}$ curves for the samples in the cast state has larger specific heat than that in the crystallized state, and the $C_{\mathrm{p}}$ in these two states all increase with the temperature rising. In addition, the specific heat differences are not obvious up to $4 \mathrm{~K}$ in their total specific heat curves.

Figure 3 shows the $C_{\mathrm{p}} / T$ versus $T^{2}$ plots for $\left(\mathrm{Fe}_{0.5} \mathrm{Co}_{0.5}\right)_{72} \mathrm{~B}_{20} \mathrm{Si}_{4} \mathrm{Nb}_{4}$ alloys in the cast state and crystallized state. In this figure, on one hand, it can be seen that the data points all fall apparently on straight lines over the range of $20 \mathrm{~K}^{2} \leq T^{2} \leq 100 \mathrm{~K}^{2}$ for $\left(\mathrm{Fe}_{0.5} \mathrm{Co}_{0.5}\right)_{72}$ $\mathrm{B}_{20} \mathrm{Si}_{4} \mathrm{Nb}_{4}$ amorphous alloy. We analyze the low temperature specific heat of $\left(\mathrm{Fe}_{0.5} \mathrm{Co}_{0.5}\right)_{72}$ $\mathrm{B}_{20} \mathrm{Si}_{4} \mathrm{Nb}_{4}$ amorphous alloy and fit it using a polynomial, and the arbitrary combinations of four terms $\left(A, T, T^{2}\right.$, and $T^{3}$, where $A$ is a constant) are considered in the fitting procedure. It is found that combination of $T$ and $T^{3}$ terms is a unique, reasonable, and accurate fit (correlation $\left.R^{2}=0.99635\right)$, while the other terms are unreasonable fit for

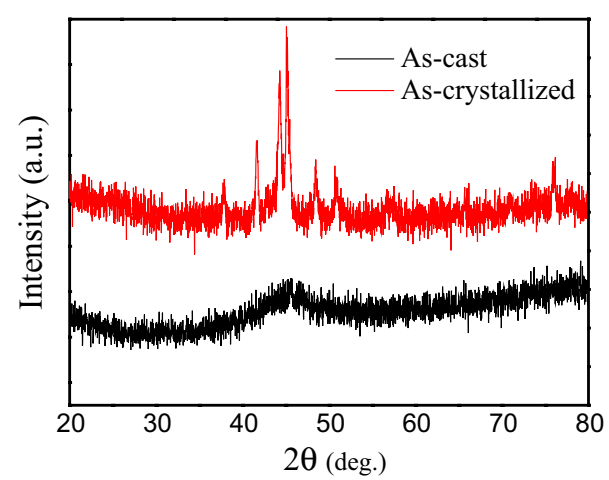

Fig. 1 XRD patterns of $\left(\mathrm{Fe}_{0.5} \mathrm{CO}_{0.5}\right)_{72} \mathrm{~B}_{20} \mathrm{Si}_{4} \mathrm{Nb}_{4}$ alloys in the cast state and crystallized state

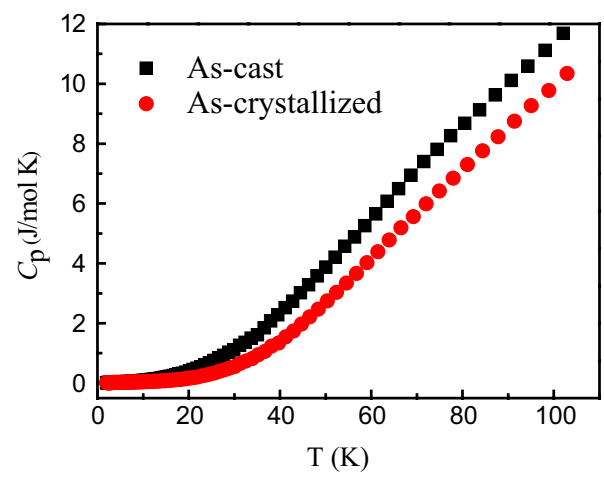

Fig. 2 The $T$-dependent $C_{p}$ for $\left(\mathrm{Fe}_{0.5} \mathrm{CO}_{0.5}\right)_{72} \mathrm{~B}_{20} \mathrm{Si}_{4} \mathrm{Nb}_{4}$ alloys in the cast state and crystallized state 


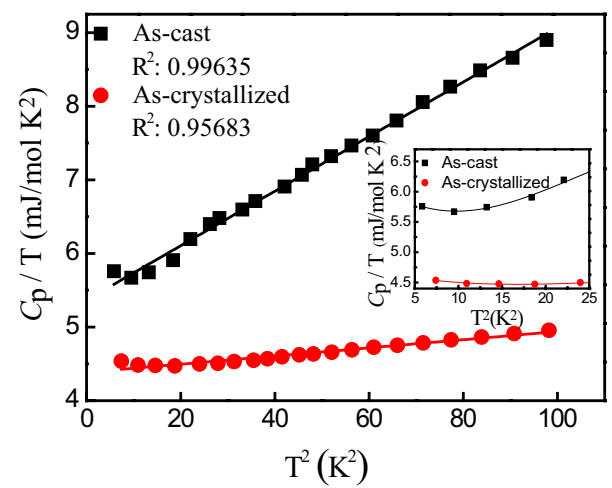

Fig. 3 The $C_{p} / T$ versus $T^{2}$ for $\left(\mathrm{Fe}_{0.5} \mathrm{Co}_{0.5}\right)_{72} \mathrm{~B}_{20} \mathrm{Si}_{4} \mathrm{Nb}_{4}$ alloys in the cast state and crystallized state. The solid lines are linear square fits of the data. The inset is the enlarged plot for $T^{2} \leq 25 \mathrm{~K}^{2}$

samples. For example, a polynomial fit to the specific heat of amorphous alloy with form of $C_{\mathrm{p}}=A_{0}+A_{1} T+A_{2} T^{2}+A_{3} T^{3}$ produces negative values of the coefficients $A_{1}$ and $A_{3}$, which are obviously unreasonable in physics. Similar problem exists in fit by combination of $T, T^{2}$, and $T^{3}$. Fitting is also unacceptable by only one term of $T^{3}$, which is in agreement with the previous report (Bai et al. 2002). Moreover, the combination of $T$ and $T^{3}$ terms obtained is applied in the fitting and compared with previous experimental data, as shown in Fig. 4a, b. So, it is reasonable to employ the fitting expression $C_{\mathrm{p}}=\gamma T+\delta T^{3}$ (where $\gamma, \delta$ are constant, and $\gamma=A_{1} ; \delta=A_{3}$ ) with different $\gamma$ and $\delta$ values, and Table 1 shows the fitting parameters of the specific heats. On the other hand, from Figs. 3 and 4, we can see that when $T^{2} \leq 20 \mathrm{~K}^{2}$, the experimental data all are slightly deviated from the linear fitting results, and represents a boson peak, respectively, in $C_{\text {phonon }} / T^{3}$ versus $T$ curves, which has been reported in detail (Zheng 1987; Shintani and Tanaka 2008; Hou et al. 2015). The inset of Fig. 3 shows the enlarged plot for $T^{2} \leq 25 \mathrm{~K}^{2}$, and when $T^{2} \leq 20 \mathrm{~K}^{2}$, it shows an obvious curve. Thus, we study the specific heat data when $T \geq 4.5 \mathrm{~K}$ and the fitting expression is reasonable.

From Table 1, one can see that $\gamma$ value is changed from 5.366 to $4.385 \mathrm{~mJ} /\left(\mathrm{mol} \cdot \mathrm{K}^{2}\right)$ with annealing from cast to crystallized state for $\left(\mathrm{Fe}_{0.5} \mathrm{Co}_{0.5}\right)_{72} \mathrm{~B}_{20} \mathrm{Si}_{4} \mathrm{Nb}_{4}$ alloy. In order to explore whether this change is universal, we choose $\mathrm{Zr}_{41.2} \mathrm{Ti}_{3.8} \mathrm{Cu}_{12.5} \mathrm{Ni}_{10} \mathrm{Be}_{22.5}$ (Zhang et al. 2001), $\mathrm{Zr}_{52.5} \mathrm{Cu}_{17.9} \mathrm{Ni}_{14.6} \mathrm{Al}_{10} \mathrm{Ti}_{5}$ (Hou et al. 2015), $\left(\mathrm{Cu}_{50} \mathrm{Zr}_{50}\right)_{90} \mathrm{Al}_{7} \mathrm{Gd}_{3}$ (Li et al. 2006, 2008), $\left(\mathrm{Cu}_{50} \mathrm{Zr}_{50}\right)_{96} \mathrm{Al}_{4}$ (Li et al. 2008), $\left(\mathrm{Cu}_{50} \mathrm{Zr}_{50}\right)_{92} \mathrm{Al}_{8}$ (Li et al. 2008) and $\mathrm{Cu}_{50} \mathrm{Zr}_{50}$ (Tang et al. 2005) alloys to obtain relevant parameters $\gamma, \delta$ by fitting their low temperature specific heats, and the corresponding Debye temperature $\theta_{\mathrm{D}}$ is calculated by $\delta=\frac{12 \pi^{4}}{5 \theta_{\mathrm{D}}} R$ (Huang 1988). They are also shown in Table 1. Meanwhile, Fig. 5 shows the changes of $\gamma$ for alloys in the cast state and the crystallized state, in which the solid and cross-hatched histograms represent alloys in the cast state and the crystallized state, respectively. It clearly can be seen from Table 1 and Fig. 5 that $\gamma$ value in crystallized state is smaller than that in the cast state.

The difference of $\gamma$ values in amorphous and crystallized alloys stimulates us to think over the following questions: what is the relationship between specific heats for amorphous and crystallized alloys, and how does the microstructure change affect the specific heat? Thus, theoretical analysis on the similarities and differences of specific heat 

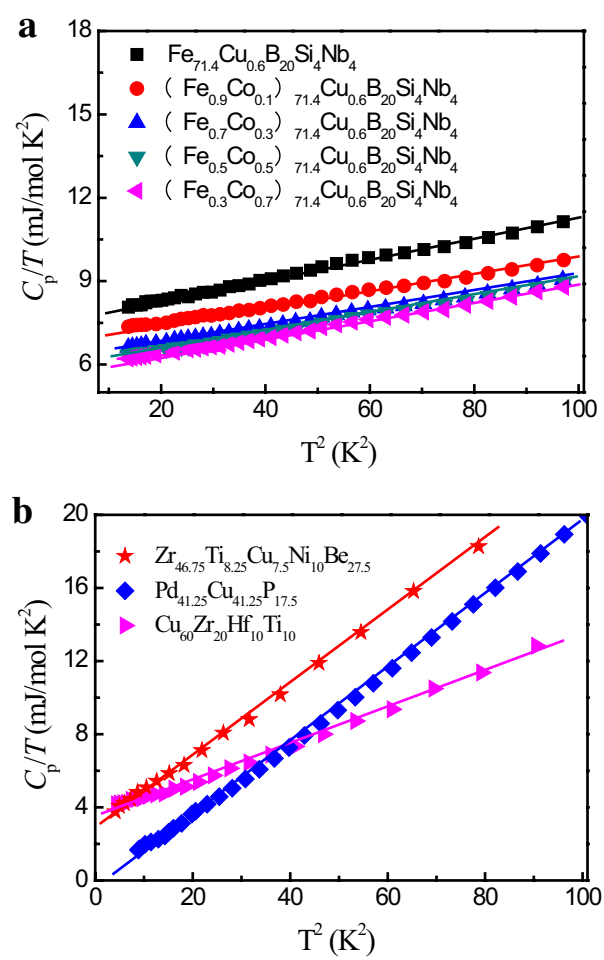

Fig. 4 The $C_{p} / T$ versus $T^{2}$ for amorphous alloys. The solid lines are linear square fits of the data. The $\mathbf{a}$ data are taken from (Yang et al. 2014a, b) and b data are taken from (Wang et al. 2011; Yang et al. 2014b; Vasiliev et al. 2009)

Table 1 Values of $\gamma, \delta$ and $\theta_{D}$ for alloys in the cast state and the crystallized (CR) state

\begin{tabular}{|c|c|c|c|c|c|}
\hline Number & Samples & States & $\gamma \mathrm{mJ} /\left(\mathrm{mol} \mathrm{K}^{2}\right)$ & $\delta \mathrm{mJ} /\left(\mathrm{mol} \mathrm{K}^{4}\right)$ & $\theta_{\mathrm{D}} / \mathrm{K}$ \\
\hline \multirow[t]{2}{*}{1} & \multirow[t]{2}{*}{$\mathrm{Zr}_{41.2} \mathrm{Ti}_{3.8} \mathrm{Cu}_{12.5} \mathrm{Ni}_{10} \mathrm{Be}_{22.5}^{\mathrm{a}}$} & Cast & 3.030 & 0.101 & 268.120 \\
\hline & & CR-805 K & 1.830 & 0.053 & 331.500 \\
\hline \multirow[t]{2}{*}{2} & \multirow[t]{2}{*}{$\mathrm{Zr}_{52.5} \mathrm{Cu}_{17.9} \mathrm{Ni}_{14.6} \mathrm{Al}_{10} \mathrm{Ti}_{5}^{\mathrm{b}}$} & Cast & 3.440 & 0.150 & 234.890 \\
\hline & & CR-973 K & 3.170 & 0.077 & 293.360 \\
\hline \multirow[t]{2}{*}{3} & \multirow[t]{2}{*}{$\left(\mathrm{Cu}_{50} \mathrm{Zr}_{50}\right)_{90} \mathrm{Al}_{7} \mathrm{Gd}_{3}^{\mathrm{c}}$} & Cast & 4.900 & 0.210 & 209.820 \\
\hline & & CR-520 K & 4.770 & 0.200 & 213.260 \\
\hline \multirow[t]{2}{*}{4} & \multirow[t]{2}{*}{$\left(\mathrm{Cu}_{50} \mathrm{Zr}_{50}\right)_{96} \mathrm{Al}_{4}^{\mathrm{d}}$} & Cast & 3.700 & 0.180 & 220.880 \\
\hline & & CR-490 K & 3.461 & 0.165 & 227.380 \\
\hline \multirow[t]{2}{*}{5} & \multirow{2}{*}{$\left(\mathrm{Cu}_{50} \mathrm{Zr}_{50}\right)_{92} \mathrm{Al}_{8}^{\mathrm{e}}$} & Cast & 3.060 & 0.170 & 225.290 \\
\hline & & CR-673 K & 2.970 & 0.100 & 268.880 \\
\hline \multirow[t]{2}{*}{6} & \multirow[t]{2}{*}{$\left(\mathrm{Fe}_{0.5} \mathrm{Co}_{0.5}\right)_{72} \mathrm{~B}_{20} \mathrm{Si}_{4} \mathrm{Nb}_{4}^{\mathrm{f}}$} & Cast & 5.366 & 0.037 & 374.540 \\
\hline & & CR-923 K & 4.385 & 0.006 & 686.830 \\
\hline \multirow[t]{2}{*}{7} & \multirow[t]{2}{*}{$\mathrm{Cu}_{50} \mathrm{Zr}_{50}^{\mathrm{g}}$} & Cast & 4.300 & 0.129 & 246.830 \\
\hline & & CR-765 K & 2.740 & 0.120 & 252.840 \\
\hline
\end{tabular}

a Zhang et al. (2001)

b Hou et al. (2015)

c Li et al. $(2006,2008)$

d,e Li et al. (2008)

f This work

${ }^{g}$ Tang et al. (2005) 


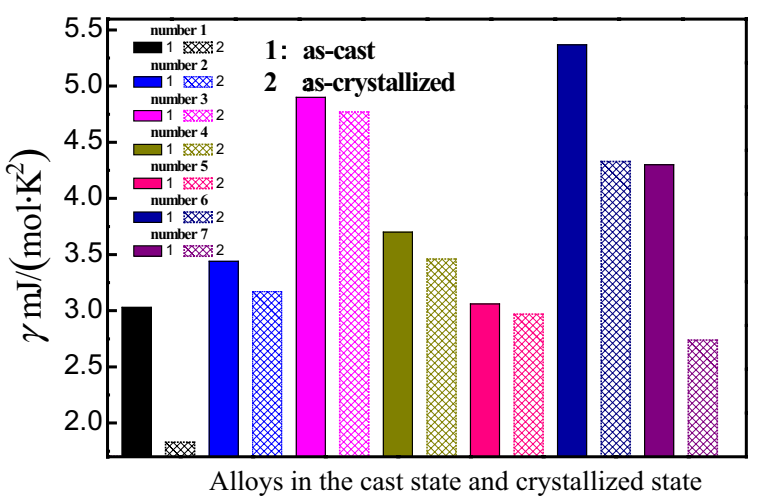

Fig. 5 The changes of electronic specific heat coefficient $\gamma$ for alloys in the cast state and crystallized state

at low temperature in two states and the effects of crystallization are provided in the following part.

\section{Specific heats for crystallized alloys}

For crystallized alloys, previous studies (Huang 1988; Luborsky 1989) have concluded that the specific heat $C_{\mathrm{p}}^{1}$ at low temperature is comprised of an electronic term and a phonon term, which can be expressed as:

$$
C_{\mathrm{p}}^{1}=\gamma_{\mathrm{c}} T+\delta_{\mathrm{c}} T^{3}
$$

where $\gamma_{\mathrm{c}}=\frac{1}{3} \pi^{2} k_{B}^{2} N\left(E_{F}^{0}\right)$ is electronic specific heat coefficient, $\delta_{\mathrm{c}}=\frac{12 \pi^{4}}{5} R\left(\frac{k_{\mathrm{B}}}{\hbar w_{\mathrm{m}}}\right)^{3}$ is phonon specific heat coefficient, $k_{\mathrm{B}}$ is the Boltzmann constant, $R$ is the gas constant, $w_{m}$ is Debye-type vibrational frequency and $\hbar$ is reduced Planck's constant. This theory is widely used for crystallized alloys, so relating discussion has not been performed in this study.

\section{Specific heats for amorphous alloys}

Compared to crystallized alloys, although amorphous alloys do not have long-range atomic order, they do have pronounced short- or medium-range order at atomic scale, so we should consider their microstructure in disordered and some extent ordered arrangements simultaneously that may affect their specific heats.

To make this issue simpler, we consider the characteristic of some extent ordered microstructure. Like crystallized alloy, the low temperature specific heat $C_{\mathrm{p} 1}$ for amorphous alloy can also be regarded as the contributions from the phonons and electrons, and amorphous alloy is also considered as a continuous elastic medium (Zhang et al. 2001), thus, according to the energy band theory (Huang 1988), the phonon contribution to specific heat can be expressed by the cubic Debye's term $C_{\mathrm{D} 1}$, i.e., $C_{\mathrm{D} 1}=\delta_{1} T^{3}$, where $\delta_{1}=\frac{12 \pi^{4}}{5} R\left(\frac{k_{\mathrm{B}}}{\hbar w_{\mathrm{m}}}\right)^{3}$ is phonon specific heat coefficient. For the electronic contribution to specific heat $C_{\mathrm{E}}^{\mathrm{e}}$, although amorphous alloys do not have long-range order, the single electron approximation theory is still applicable (Huang 1988), so the $C_{\mathrm{E}}^{\mathrm{e}}$ is also expressed as $C_{\mathrm{E}}^{\mathrm{e}}=\frac{1}{3} \pi^{2} k_{\mathrm{B}}^{2} N\left(E_{\mathrm{F}}^{0}\right) T$. In spite of this, it has been verified that the $C_{\mathrm{p} 1}$ of amorphous alloys cannot be described accurately only by the phonon and the electronic contributions for 
amorphous alloys (Wang et al. 2011; Tang et al. 2005), this theory needs some improvement. Meanwhile, the existence of random arrangement of atoms is considered to affect the low temperature specific heat $C_{\mathrm{p} 1}$ for amorphous alloy, which means an extra contribution should be included, thus, from the view of disordered microstructure, the random arrangement of atoms has contributions to the $C_{\mathrm{p} 1}$, defined as $C_{\mathrm{E}}^{\mathrm{p}}$. Previous report (Kittel 2005) has pointed out that the $C_{\mathrm{E}}^{\mathrm{p}}$ originates from low-energy excitation, which is related to two-level scattering caused by random arrangement of atoms, and the low-energy excitation is also successfully explained using the two-level scattering model (Vladar and Zawadowski 1983; Cochrane et al. 1975). Therefore, $C_{\mathrm{E}}^{\mathrm{p}}$ is written as $C_{\mathrm{E}}^{\mathrm{p}}=\frac{2 k_{B}^{2}}{3 \Delta_{0}} T$, where $\Delta_{0}$ is the maximum difference between the two energy minimum points. Thus, the low temperature specific heat for amorphous alloy can be expressed as:

$$
\begin{aligned}
C_{\mathrm{p} 1} & =C_{\mathrm{E}}^{\mathrm{e}}+C_{\mathrm{D} 1}+C_{\mathrm{E}}^{\mathrm{p}}=\frac{1}{3} \pi^{2} k_{\mathrm{B}}^{2} N\left(E_{\mathrm{F}}^{0}\right) T+\frac{12 \pi^{4}}{5} R\left(\frac{k_{\mathrm{B}}}{\hbar w_{\mathrm{m}}}\right)^{3} T^{3}+\frac{2 k_{\mathrm{B}}^{2}}{3 \Delta_{0}} T \\
& =\left[\frac{1}{3} \pi^{2} k_{\mathrm{B}}^{2} N\left(E_{\mathrm{F}}^{0}\right)+\frac{2 k_{\mathrm{B}}^{2}}{3 \Delta_{0}}\right] T+\frac{12 \pi^{4}}{5} R\left(\frac{k_{\mathrm{B}}}{\hbar w_{\mathrm{m}}}\right)^{3} T^{3}
\end{aligned}
$$

Thus, Eq. (2) can be further simplified as:

$$
C_{\mathrm{p} 1}=C_{\mathrm{E} 1}+C_{\mathrm{D} 1}=\gamma_{\mathrm{a}} T+\delta_{\mathrm{a}} T^{3}
$$

where $C_{E 1}\left(C_{E 1}=C_{\mathrm{E}}^{\mathrm{e}}+C_{\mathrm{E}}^{\mathrm{p}}\right)$ is the electronic specific heat; $\gamma_{\mathrm{a}}=\frac{1}{3} \pi^{2} k_{\mathrm{B}}^{2} N\left(E_{\mathrm{F}}^{0}\right)+\frac{2 k_{\mathrm{B}}^{2}}{3 \Delta_{0}}$; $\delta_{\mathrm{a}}=\frac{12 \pi^{4}}{5} R\left(\frac{k_{\mathrm{B}}}{\hbar w_{\mathrm{m}}}\right)^{3} ; k_{\mathrm{B}}, \hbar$, and $R$ are fundamental physical constants; and $N\left(E_{\mathrm{F}}^{0}\right), \Delta_{0}$, and $w_{\mathrm{m}}$ are the physical quantities determined by materials, respectively. Here, $\gamma_{\mathrm{a}}$ and $\delta_{\mathrm{a}}$ should be invariants for given amorphous alloys.

At low temperature, it is difficult to distinguish the contributions from the electron and from the low-energy excitation (Tang et al. 2005), so $\gamma_{\mathrm{a}}$ is usually named as total electronic specific heat coefficient.

By comparing Eqs. (1) and (3), it can be seen that there is a similarity on specific heat equation for amorphous and crystallized alloys, both of them can be represented as a sum of linear electronic term and phonon term, namely:

$$
C_{p}=\gamma T+\delta T^{3}
$$

where $C_{\mathrm{p}}$ is specific heat; $\gamma$ is electronic specific heat coefficient for amorphous and crystallized alloys; $\delta$ is phonon specific heat coefficient for amorphous and crystallized alloys. However, although this conclusion is in good agreement with the fitting model, it is important to note that $\gamma$ may have different connotation for amorphous and crystallized alloys.

From Eqs. (2), (3) and (4), we obtain the electronic term in low temperature specific heat for amorphous alloys, expressed as:

$$
\frac{1}{3} \pi^{2} k_{B}^{2}\left(1+\frac{2}{\pi^{2} \Delta_{0}}\right) N\left(E_{F}^{0}\right) T=\gamma T
$$

where the variation of $\gamma$ can reflect the changes of long-rang disordered atomic arrangement in amorphous structure (Yang et al. 2010). 
From Eq. (4), it can be seen that $\gamma$ is determined by $N\left(E_{\mathrm{F}}^{0}\right)$ and $\Delta_{0}$ at a certain temperature, and it is also known that $N\left(E_{\mathrm{F}}^{0}\right)$ and $\Delta_{0}$ are changed with the material structure. So, the change of $\gamma$ can be discussed from the variations of $N\left(E_{\mathrm{F}}^{0}\right)$ and $\Delta_{0}$. On one hand, when $N\left(E_{\mathrm{F}}^{0}\right)$ plays a leading role, $\gamma$ is mainly linked to the position of $E_{\mathrm{F}}^{0}$. For amorphous alloys, the $E_{\mathrm{F}}^{0}$ is near the maximum value in the $N\left(E_{\mathrm{F}}^{0}\right)$ curve (Cao et al. 2009). When the alloys are annealed from amorphous to crystallized state, $N\left(E_{\mathrm{F}}^{0}\right)$ decreases due to the change of $E_{\mathrm{F}}^{0}$ position (Luborsky 1989; Beck and Guntherodt 1983). For transition metalmetalloid amorphous alloys, Chen et al. (1975) has pointed out that the electrons may transfer from the metalloid elements to fill the " $d$ " shells of transition metal elements, causing the change of electronic density of states. In transition metal elements systems, the " $d$ " shell has not been filled. From the perspective of energy band theory, when the amorphous alloy is formed, the less overlap of " $d$ " orbitals between elements results in a narrow band, however, there are 5 " $d$ " orbitals in transition metal element, which can make the band staggered, overlapping, result in the increment of $N\left(E_{\mathrm{F}}^{0}\right)$ in " $d$ " band (Huang 1988). When the alloy is transformed from amorphous state into crystallized state, the electrons near the Fermi surface can obtain sufficient energy, causing the electrons shift outside the Fermi surface. Thus, it results in the decrease of $N\left(E_{\mathrm{F}}^{0}\right)$ in " $d$ " band, and $\gamma$ is also reduced. In addition, the volume $V$ is normally reduced during the alloy crystallization (Kittel 2005). With the constant number of electrons $N$, it can be drawn from the density of states formula $N\left(E_{\mathrm{F}}^{0}\right)=\frac{3 m^{*} N}{h^{2}}\left(\frac{V}{3 \pi^{2} N}\right)^{2 / 3}$ ( $h$ is the Planck's constant and $m^{*}$ is the effective mass of electrons) (Kittel 2005) that $N\left(E_{\mathrm{F}}^{0}\right)$ and its corresponding $\gamma$ decrease. On the other hand, when the amorphous alloy is formed from liquid state by rapid quenching, the oversized cage structure, large voids or enough large free volume may be kept (Hirata et al. 2011), where the densities for most amorphous alloys are about $0.5-3$ \% less than those for corresponding crystallized alloys. In this kind of structure, some solute atoms are loose or weakly bounded in the interstitial intercluster sites and the vibration of these loose "rattler" atoms shows a higher frequency compared with other atoms in amorphous structure. Therefore, due to the reinforced vibration energy, the probability of the tunneling effect will be increased once the atoms overcome the potential energy barrier, which means a relative decrease of $\Delta_{0}$, and this will cause the increment of $\gamma$. Meanwhile, due to the existence of oversized cage structure in amorphous alloy, the electronic scattering in the process of transmission is enhanced, and it will result in the increase of $m^{*}$ ( $m^{*}$ is effective mass of electrons) (Zhang et al. 2001). Thus, $N\left(E_{\mathrm{F}}^{0}\right)$ and its corresponding electronic specific heat coefficient $\gamma$ increase. In conclusion, the increment of electronic density of states at the Fermi level and the vibration frequency of localized loose "rattler" atoms in the oversized cage structure are responsible for the enhancement of $\gamma$, and result in a larger electronic contribution term for amorphous alloys.

In addition, it can also be seen from Table 1 that phonon specific heat coefficient $\delta$ is decreased and Debye temperature $\theta_{\mathrm{D}}$ is increased during annealing from the cast to crystallized state, and this phenomenon can be discussed using harmonic vibration model. It is known to all that the atomic vibration frequency $w=(k / m)^{1 / 2}(k$ is the restitution coefficient and $m$ is the mass of the oscillator). When alloys are annealed from cast to crystallized state, the $k$ and its corresponding $w$ become larger, moreover, the 
corresponding $w_{\mathrm{m}}$ becomes larger (Huang et al. 2014; Bai et al. 2001). According to the Debye temperature $\theta_{\mathrm{D}}=\hbar w_{\mathrm{m}} / k_{\mathrm{B}}$ and $\delta=\frac{12 \pi^{4}}{5} \times \frac{R}{\theta_{\mathrm{D}}^{3}}, \theta_{\mathrm{D}}$ is increased and $\delta$ is decreased.

\section{Conclusions}

The low temperature specific heats of $\left(\mathrm{Fe}_{0.5} \mathrm{Co}_{0.5}\right)_{72} \mathrm{~B}_{20} \mathrm{Si}_{4} \mathrm{Nb}_{4}$ amorphous and corresponding crystallized alloys have been investigated. It is demonstrated that the cast state for amorphous alloy has larger specific heat compared with its crystallized state at low temperature. Meanwhile, it is also found that the electronic contribution term for alloy in cast state is larger than that in the crystallized state, and this universal phenomenon is interpreted using the electronic density of states and localized harmonic modes based on the vibrations of loose "rattler" atoms in oversized cage structure. The result has important significance for understanding the effect mechanism before and after crystallization on the electronic specific heat for amorphous alloys at low temperature.

\section{Authors' contributions}

$\mathrm{LH}$ and $\mathrm{HL}$ conceived of the study and the experimental design and contributed to the manuscript. JM, QL and BS contributed to the experiments, and MY contributed to the data analysis. All authors read and approved the final manuscript.

\section{Author details}

1 School of Sciences, School of Mechanics and Civil Engineering, State Key Laboratory for Geomechanics and Deep Underground Engineering, China University of Mining and Technology, Xuzhou 221116, China. ${ }^{2}$ School of Materials Science and Engineering, Southeast University, Nanjing 211189, China.

\section{Acknowledgements}

This work was supported by the Fundamental Research Funds for the Central Universities (2014ZDPY35).

\section{Competing interests}

The authors declare that they have no competing interests.

Received: 31 March 2016 Accepted: 11 May 2016

Published online: 23 May 2016

\section{References}

Bai HY, Luo JL, Chen ZJ, Wang WH (2001) Low temperature specific heat of bulk glassy and crystalline $\mathrm{Zr}_{41} \mathrm{Ti}_{14} \mathrm{Cu}_{12.5} \mathrm{Ni}_{1}$ ${ }_{0} \mathrm{Be}_{22.5}$ alloys. Appl Phys Lett 78(18):2697-2699

Bai HY, Luo JL, Zhang J, Chen ZJ (2002) Low temperature specific heat of a typical glass forming alloy. J Appl Phys 91(11):9123-9127

Beck H, Guntherodt HJ (1983) Glassy metals II. Springer, Berlin

Blázquez JS, Millán M, Conde CF, Franco V, Conde A, Lozano-Pérez S (2008) Nanocrystallization effects on the specific heat of Fe-Co-Nb-B amorphous alloy. J Non-Cryst Solids 354(354):5135-5137

Burin AL (1995) Dipole gap effects in low energy excitation spectrum of amorphous solids. Theory for dielectric relaxation. Czech J Phys 100(3):309-337

Cao LZ, Yan SS, Chen ZJ (2009) Low-temperature Physics II. Press of Science and Technology University in China, Hefei

Chen HS, Krause JT, Coleman E (1975) Elastic constants, hardness and their implications to flow properties of metallic glasses. J Non-Cryst Solids 18(2):157-171

Cochrane RW, Harrs R, Stroolson JO, Zuckermann MJ (1975) Structural manifestations in amorphous alloys: resistance minima. Phys Rev Lett 35:676-679

Dun CC, Liu HS, Hou L, Xue L, Dou LT, Yang WM, Zhao YC, Shen BL (2014) Ductile Co-Nb-B bulk metallic glass with ultrahigh strength. J Non-Cryst Solids 386(3):121-123

Grace JM, Anderson AC (1989) Low-temperature specific heat and thermal conductivity of a glassy polymer under applied pressure. Phys Rev B 40(40):1901-1917

Hirata A, Guan P, Fujita T, Hirotsu Y, Inoue A, Yavari AR, Sakurai T, Chen M (2011) Direct observation of local atomic order in a metallic glass. Nat Mater 10(1):28-33

Hou L, Liu HS, Liu QL, Dun CC, Yang WM, Huo JT, Dou LM (2015) Effects of crystallization on boson peak of $\mathrm{Zr}_{52.5} \mathrm{Cu}_{179} \mathrm{Ni}_{146} \mathrm{Al}_{10} \mathrm{Ti}_{5}$ bulk metallic glass. J Low Temp Phys 178(1-2):11-17

Huang K (ed) (1988) Solid physics. Higher Education Press, Beijing, pp 149-154, 213-224, 276-286

Huang B, Bai HY, Wang WH (2014) Relationship between boson heat capacity peaks and evolution of heterogeneous structure in metallic glasses. J Appl Phys 115(15):153505-153505-7

Kanomata T, Sato Y, Sugawara Y, Kimura HM, Kaneko T, Inoue A (2008) Specific heat of Zr-based metallic glasses. J Alloys Compd 461(1-2):39-41 
Kittel C (ed) (2005) Introduction to solid state physics. Chemical Industry Press, Beijing, pp 391-392

Kroeger DM, Koch CC, Scarbrough JO, McKamey CG (1984) Effects of short-range order on electronic properties of Zr-Ni glasses as seen from low-temperature specific heat. Phys Rev B 29(3):1199-1208

LiY, Bai HY, Wang WH, Samwer K (2006) Low-temperature specific-heat anomalies associated with the boson peak in CuZr-based bulk metallic glasses. Phys Rev B 74(5):2201

Li Y, YU P, Bai HY (2008) Study on the boson peak in bulk metallic glasses. J Appl Phys 104(1):699-703

Liu WL, Wang YG, Chen FG (2014) Effect of Ti on glass-forming ability and magnetic properties of $\mathrm{Fe}_{81} \mathrm{Si}_{4} \mathrm{~B}_{12-x} \mathrm{P}_{2} \mathrm{Cu}_{1} \mathrm{Ti}_{x}$ $(x=0-3)$ soft magnetic alloys. J Mater Sci Mater Electron 25(11):5066-5070

Luborsky FE (1989) Amorphous metallic alloys. Metallurgical Industry Press, Beijing, pp 617-618

Machida K, Ichioka M (2008) Magnetic field dependence of low-temperature specific heat in $\mathrm{Sr}_{2} \mathrm{RuO}_{4}$. Phys Rev B 77(18):998-1002

Mikla VI, Mikla WV (2009) Structural transformations in amorphous As $\mathrm{Se}_{1-x}(0 \leq x \leq 0.20)$ films. J Mater Sci Mater Electron 20(11):1095-1105

Shintani H, Tanaka H (2008) Universal link between the boson peak and transverse phonons in glass. Nat Mater $7(11): 870-877$

Tang MB, Bai HY, Wang WH (2005) Tunneling states and localized mode in binary bulk metallic glass. Phys Rev B 72(1):2202

Vasiliev AN, Voloshok TN, Granato AV, Joncich DM, Mitrofanov YuP, Khonik VA (2009) Relationship between low-temperature boson heat capacity peak and high-temperature shear modulus relaxation in a metallic glass. Phys Rev B 80(17):2102

Vladar K, Zawadowski A (1983) Theory of the interaction between electrons and the two-level system in amorphous metals. I. Noncommutative model Hamiltonian and scaling of first order. Phys Rev B 28(3):1564-1581

Wang SY, Wang CZ, Li MZ, Huang L, Ott RT, Kramer MJ, Sordelet DJ, Ho KM (2008) Short- and medium-range order in a $\mathrm{Zr}_{73} \mathrm{Pt}_{27}$ glass: experimental and simulation studies. Phys Rev B 78(18):184204

Wang ZX, Sun B, Lu JB (2011) Effects of crystallization on low-temperature specific heat capacity of $\mathrm{Cu}_{60} \mathrm{Zr}_{20} \mathrm{Hf}_{10} \mathrm{Ti}_{10}$ bulk metallic glass. Trans Nonferr Met Soc Chin 21(6):1309-1313

Wang JQ, Liu YH, Chen MW, Xie GQ, Louzguine-Luzgin DV, Inoue A, Perepezko JH (2012) Rapid degradation of Azo Dye by Fe-based metallic glass powder. Adv Funct Mater 22(12):2567-2570

Xiang R, Zhou SX, Dong BS, Zhang GD, Li ZZ, Wang YG (2014) The excellent soft magnetic properties and corrosion behaviour of nanocrystalline FePCCu alloys. J Mater Sci Mater Electron 25(7):2979-2984

Xiang R, Zhou SX, Dong BS, Zhang GD, Li ZZ, Wang YG (2015) Role of Co on microstructure, crystallization behavior and soft magnetic properties of $\left(\mathrm{Fe}_{1-x} \mathrm{CO}_{x}\right)_{84} \mathrm{Si}_{4} \mathrm{~B}_{8} \mathrm{P}_{3} \mathrm{Cu}_{1}$ nanocrystalline alloys. J Mater Sci Mater Electron 26(4):2076-2081

Yang WM, Liu HS, Yang XQ, Dou LM (2010) Low temperature specific heat of amorphous alloys. J Low Temp Phys 160(3-4):148-155

Yang WM, Liu HS, Dun CC, Li JW, Zhao YC, Shen BL (2013) Nearly free electron model to glass-forming ability of multicomponent metallic glasses. J Non-Cryst Solids 361(2):82-85

Yang WM, Zhao YC, Dou L, Dun CC, Zhang J, Li M, Zhao G, Xue L, Bian HT, Liu HS (2014a) Correlation between fractal dimension and strength of brittle bulk metallic glasses. Mater Sci Tech 30(4):447-450

Yang WM, Liu HS, Liu XJ, Chen GX, Dun CC, Zhao YC, Man QK, Chang CT, Shen BL, Inoue A, Li RW, Jiang JZ (2014b) Correlation of atomic packing with the boson peak in amorphous alloys. J Appl Phys 116(12):123512-123512-5

Yu HB, Wang WH, Bai HY, Wu Y, Chen MW (2010) Relating activation of shear transformation zones to $\beta$ relaxations in metallic glasses. Phys Rev B 81(22):220201

Zhang J, Luo JL, Bai HY, Wang WH, Chen ZJ, Meng JB, Wang YP, Lin DH, Tong CZ, Jin D (2001) Low temperature specific heat on bulk amorphous Zr-Ti-Cu-Ni-Be alloy. Acta Phys Sin 50(9):1747-1750

Zheng ZB (ed) (1987) Amorphous solid materials. Science Press, Beijing, pp 375-380

Zhou ZH, Uher C, Xu DH, Johnson WL, Gannon W, Aronson MC (2006) On the existence of Einstein oscillators and thermal conductivity in bulk metallic glass. Appl Phys Lett 89(3):031924-031924-3

\section{Submit your manuscript to a SpringerOpen ${ }^{\circ}$ journal and benefit from:}

- Convenient online submission

- Rigorous peer review

- Immediate publication on acceptance

- Open access: articles freely available online

- High visibility within the field

- Retaining the copyright to your article

Submit your next manuscript at $\boldsymbol{\nabla}$ springeropen.com 